\section{REFLECTOR AND PORTRAIT LENS IN CELESTIAL PHOTOGRAPHY.}

$\mathrm{T}^{\mathrm{T}}$ has been much discussed recently, whether the reflector is preferable to a portrait lens in celestial photography. It may be known to the readers of NATURE that I am interested in this subject, since I believe I was the first to use the portrait lens for the purpose of seeking for large and wide-spread nebulæ.

No doubt the reflector has many advantages over the portrait lens--advantages which Dr. Roberts has often dwelt upon. In consequence of the small absorption of light, the lack of the different surfaces, and the absolute correction for chemical rays, the focal pictures with the reflector must theoretically be much better than with the doublet. The two last-named points are especially effective; for the star discs are made much smaller and increase much slower than with the portrait lens. I have shown absorption of light in the glass of the lens, in the latter the discs of images are larger, and therefore not so intense. But the reflecting power of the mirror is always soon diminished through the influence of oxidation, and therefore the reflector does not surpass the portrait lens practically as much as would be expected.

The advantages which exist for small reflectors over small portrait lenses increase accordingly to the diameters; the absorption in the portrait lens becomes rapidly greater and the sharpness less, and thus the large mirror will surpass encore plus the large doublet.

In spite of this, the portrait lens is much superior to the reflector for the work of seeking and charting feeble and extended nebulosities.

The lens takes a much larger field of the sky than the mirror. This is known to be the reason why the portrait lens should be used exclusively for the photography of minor planets, of comets, for making charts, and especially

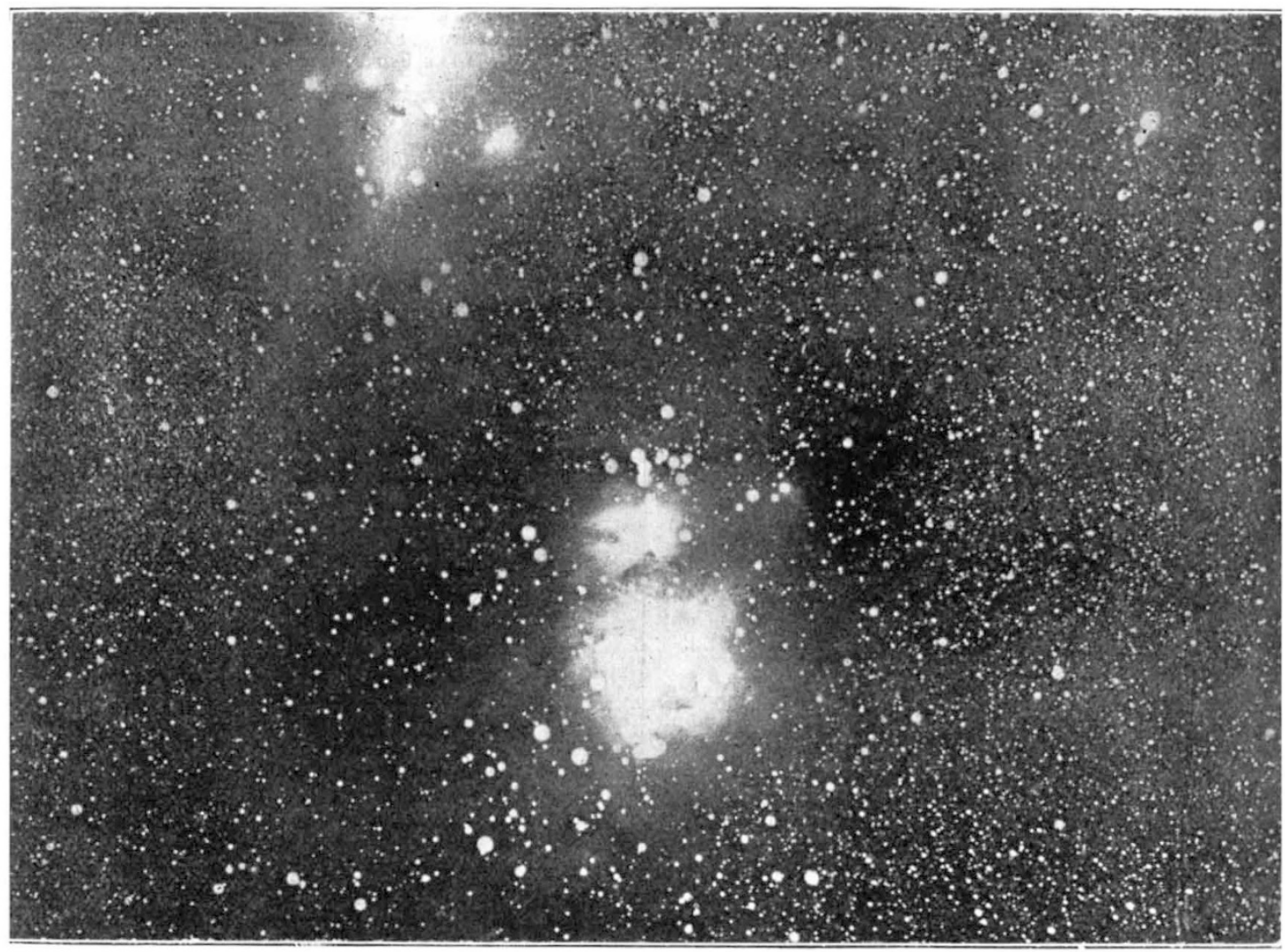

FIG. r.-The nebula of Orion.

that the lenses themselves are the reason for the increase of star discs in the film (Photogr. Corresp., 1892), therefore I fully understand the advantages of the reflector.

With my 6-inch Voigtländer portrait lens, the discs of the smallest stars in the Milky Way have a diameter of 6 to 8 seconds of arc; I think with a good reflector such discs may have a much smaller diameter.

I do not know the diameter of the smallest star discs on plates taken with Dr. Roberts' 20 -inch reflector; we may believe, however, that in his best pictures the sharpness of the image is much greater than with my 6-inch portrait lens.

As to the light-gathering power--regarding the proportion of aperture to focal length the same in the two cases-there are two reasons why it is greater with the reflector than with the portrait lens. Besides the greater or general views of the Milky Way. This last point has been taken up (Monthly Notices, R.A.S., vol. lvii. No. I) by Prof. Barnard against Dr, Roberts. Barnard shows that the portrait lens is far better adapted to give the general structure of the Milky Way than the mirror, in which the field is so small.

I was sure of this from the beginning, and afterwards all my plates showed that there is still another reason for preferring the portrait lens to the reflector; a reason which, depending likewise upon the large field, would alone have decided me to use even a big portrait lens instead of a reflector.

When photographing regions of sky covered with feeble and extended nebulosities, only feeble and extended darker parts are obtained upon the plate. These can only be seen if there are besides these parts othes 


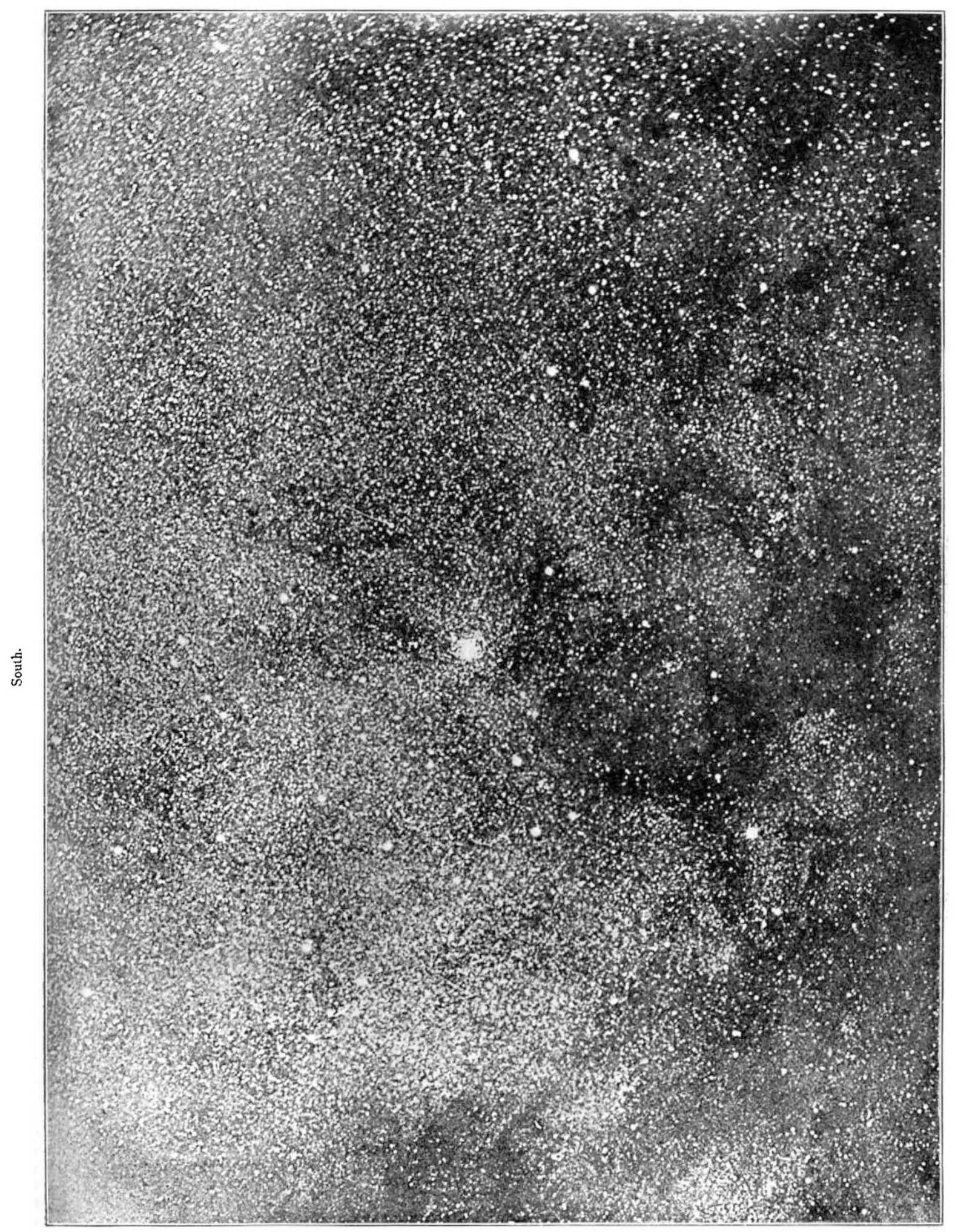

parts on the plate free from nebulous matter, and therefore appearing lighter.

The nebulosities only are clearly visible, if the borders between nebula and dark sky are followed across a large surface. It follows that this can be done only with large fields, and will never be possible with the reflector; therefore, in this case, the portrait lens is preferable to the reflector. NO. I 434 , VOL. 55$]$ 
With a mirror I should not have found the large nebulæ in Cygnus, the large nebulosities of Taurus, of Cassiopeia, of Aquila, of Orion. and of many other regions. I only could find them on the ample field of the portrait lens.

The very small doublets-for instance the small lantern Ienses, which have been used by myself, and afterwards by Prof. Barnard-are in certain cases inferior to larger lenses. The smaller lenses crowd the nebulous masses, found the nebulosities in Taurus, and the America nebula for the first time, on plates which were exposed for only a very short time. A second way is to use a mono. chromatic light to examine the plates then slight differences in brightness can be easily seen.

Orion was a good subject for the finding of such diffused nebulæe. Besides the well-known nebulæ around $\zeta$ and $\theta$ Orionis, and besides the many small nebulæ, there are feeble and widespread nebulosities, some of which have been discovered by Profs. $W^{2}$. Pickering, Barnard, and the writer, with the aid of portrait lenses. These would still be unknown but for the portrait lens, because a plate taken with a reflector would cover only a minute part of the nebulosities, and there would be no parts free from nebulosity in this small field, to be compared with the nebulous parts.

I may here remark that I discovered easily the interesting connection between the nebulre of $\theta$ Orion and $\zeta$ Orion (Fig. 1 ) This seems a most important example of the connection of tivo large and far distant nebula; and because the connection is effected over a wide field, it promises to bring new light on the knowledge of the situation of the nebulæe in the universe Fig. I is a slightly enlarged print of a plate of this part of the sky, taken with a 6-inch Voigtländer lens of 30 -inch focus.

The broad long-spread train of nebulosity, which appears in this marvellous bay running from $\zeta$ Orionis nearly in a straight line against S.S.E., becomes broader and broader growing southwards, and at the same time fainter and fainter. It makes a wide curve and runs much more south than the brighter parts of the $\theta$ Orion nebula. Now, in every direction we see streams of nebulosity running from north, east, and south from the $\zeta$ nebula to the $\theta$ nebula. Especially from the north come down many lacerated and finelydrawn ribbons of nebulosity connecting the $\zeta$ Orion nebula with the $\theta$ nebula. The most marvellous connection is by the above-named broad stream, which runs much more south than the $\theta$ nebula, and passes over to it from south-east in a large arch.

Besides these nebulæ lie very extended relatively bright nebulosities to the west of the $\theta$ Orion nebula, connected with it by

hide the rifts, and consequently make the trains of nebulosity less clear than a slightly larger portrait lens would do.

If the plates are not exposed long enough, various devices have to be employed to discover the faint nebulosities on the plate. One simple way of doing this, is to press the plate in a printing-frame upon smooth white paper, and to examine it in the sunlight. In this way I many streams ; and also mighty nebulosities are situated
to the east of the circular train, which comes down from $\zeta$ Orion nebula. These are likewise connected together at almost every possible place.

The picture is a beautiful and marvellous one, yet we see only the roughest connections and streams.

It needs long-sustained efforts and much work before we shall be able to know this region, so that we can 
reproduce the pictures of the portrait lens as a chart of it.

This is an example of what portrait lenses have given us in quite a new direction. If we find out, as in this case, that two such nebule are connected in spite of their being far distant on the sky, this gives us the impulse for quite a new comprehension of the universe; and all the theories of this kind have to be given up in favour of a new one.

I gave other examples of such large nebulæ several years ago in Knowledge, and perhaps they are still in the memory of some of the readers.

An example of the connection of a cluster with the Milky Way, and the general structure of a part of the same, is given in Fig. 2. The cluster is "Messier II, in Scutum, exposure $3 \frac{1}{4}$ hours, enlarged part of the original plate. It will be seen at first sight, that those mighty masses of faint stars and star dust show the most interesting stratifications and connections. The cluster looks like the centre of a moved system. We see here at a glance, that it would have been impossible to get such views of star streams with the small field of a reflector.

I use this opportunity to state that I made last year several inprovements in finding and reproducing nebulous masses, using a method of strengthening the image by reprinting it. It is possible - by printing the original plate successively on otherplates - to reinforce the feeble contrasts between the nebulosities and the background of sky, so that one can see at a glance such nebule on the reproduction, of which the traces are only to be suspected on the original plate. It looks very curious to see side by side the original and the reproduced negative. My friend Prof. Naegamvala, to whom I communicated the method last year, has published something about this matter in the Journal of the British Astronomical Association (vol. vii. No. 3). $\mathrm{He}$ and $\mathrm{Mr}$. Lunt had great success with the nebula M.8, using this method. The reprinting ought to be done upon slow plates in over-exposing, and, in developing, very slow developers should be used.

I give here (Fig. 3 ) the twice reprinted reproduction of a plate of the Andromeda nebula, as an example of the results obtained. The original (of four hours' ex. posure with a 5 -inch aplanatic lens, made in 1891 ) was very faint, so that it would have been impossible to make a good print of it. It is not possible to go further on in this way, because the grain becomes troublesome. But using transferrotype-collodion-paper or dry-collodionplates, the process can be repeated several times. The process used for reproduction is unable to make a satisfactory comparison between the first and second NO. I 434 , VOL. 55$]$ reproduction of the nebula, so only one illustration is given.

For another example I give the outer-nebula of the great Orion nebula $(\theta)$, which are reproduced here the first time (Fig. 4). We see many interesting streams of nebulosity all around, which never thus can be given at once by a reflector. The print is from an enlargement of a negative of $4 \frac{1}{2}$ hours' exposure, with a 6 -inch portrait lens.

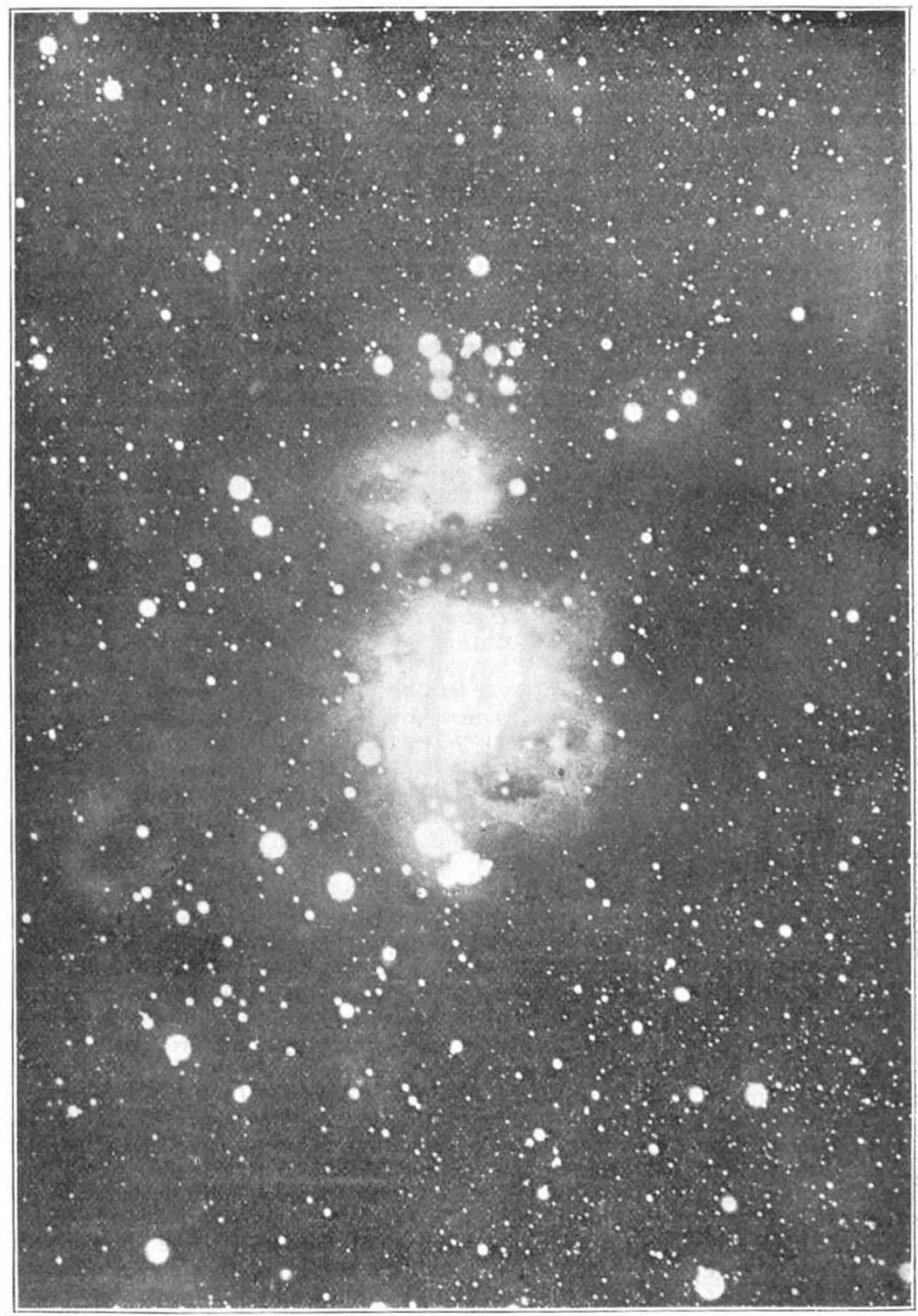

FIG. 4.-The surrounding nebulæ of the Great Orion nebula.

In spite of using this small 6-inch lens, nearly all detail visible in brighter parts photographed on Dr. Roberts' photographs is quite well visible here. It must be possible to get, within a certain limit, detailed pictures of nebulæe by portrait lenses. No doubt, a good and large reflector will give much more detail ; but the difference between reflector and portrait lens is not so great as is often supposed. The sharpness of the image of the portrait 
lens is better than is assumed by various astronomers; I found it about ten times greater than it is given, for instance, by l'rof. Wilsing in his recently-published paper in Astr. Nichr., No. 3400.

The weak point in photographing stars and nebula is not in the instrument; it is in the plate. We know that the Pleiades are surrounded by wide-spread nebulosities, shown by Prof. Barnard and myself. Now for four years 1 have been working at a chart of this nebulous region, but it has been impossible to get to an end till now. In this case the plates did not allow it ; either they were not sensitive enough, so that I got nothing, or if they were, they had streams or spots looking exactly like nebule. I made several dozens of exposures of the Pleiades, some of twelve hours' exposure, containing beautiful nebulosities, but no plate has been sufficient. All of them show, besides the true nebulosities, more or less artificial nebulx, making it impossible to find out exactly the structure of the true nebulosities. Thus we need often a large number of plates to get the true nebulosities ready for charting. This is now the chief question for celestial photography.

Photographs of small nebulac taken with portrait lenses often show much detail ; for instance, the nebulic near $\gamma$ Cassiopeice, called by Barnard the "fan-shaped" nebulic. These nebulæ were photographed the first time

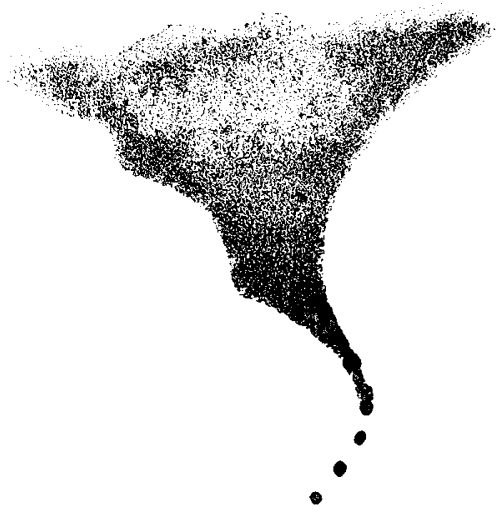

FIt;. 7.-Fan-shaped nebula in Cassiopeia.

by myself, December 30, I893, and described in the Astr. Nichr., No. 32 I4. Prof. Barnard obtained them several weeks later, and he showed that these nebulic represent a good example for the advantages of portrait lenses over reflectors in discovering nebulæ, because Dr. Roberts did not find them on his plates. Now these nebulia seem to me of the greatest importance for the comprehension of the genesis of stars; and especially for the theories of Mr. Lockyer, these will be found very interesting objects. Several years ago I gave in Astr. Nachr., No. 3217 , an illustration of one of those nebulae $\left[\mathrm{o}^{\mathrm{h}} 52^{\mathrm{n}}+60^{\circ} 5^{\prime}(\mathrm{I} 860)\right]$, and the sketch is reprinted here (Fig. 5). I have shown that the nebula looks like a tornado, in the concentrated part of which the stars are formed, and that thus the chain formed by the stars may be understood.

There we have the point where our small portrait lenses fail, and where the reflector finds its place. The lens has found the nebula, and given the first idea of its constitution; but the large mirror will bring out here the details necessary for our knowledge. It is the same as with the small spiral nebulie, of which Dr. Roberts' plates have shown us the true form.

To me it is quite incomprehensible how it was possible to begin a dispute about the use of the portrait lens in celestial photography. The portrait lens has given us so much, that it is now too late to discuss its efficiency.
The doublet finds the nebula ... I will not speak of contets, planets, \&c..-and throws light upon the ways in which the large nebulous streans spread over enormous parts of the sky. The charting and following of these streams forms now one of the most important problems of astronomy. Therefore this instrument is absolutely necessary for us. It brings us also to a certain high degree of knowledge of the finer detail, though not nearly so high as the mirror. But with portrait lenses not too large we can expose very long, and over several nights, so that we can get traces of nebulce and stars, which we can never find with the large reflector, because such very long exposures are not quite possible with a reflector, for technical reasons.

For these points the reflector has to recede. It is true the portrait lens will often photograph certain objects as nebulous, which will be found later formed by smallest stars. but the pictures of the reflector show, likewise, at many places nebulosities which, I am sure, are composed of relatively bright stars. An example of this effect has been given by Prof. Barnard, for the case of Dr. Roberts' plates (Monthly Notices, lvii. No. 1). The difference between the two instruments in this direction is not a very great one, and because the portrait lens is absolutely necessary to us in so many problems shown here, we have to use it as often as we can for these purposes, and to leave the reflector to work out the finest details of special points. MAX Wor.F.

Heidelberg, Astrophys. Observatory, March 1897.

\section{THE TIEEVTYFIFTH ANNIVERSARY OF THE FOUNDATION OF THE NAPLES ZOOLOGICAL STATION.}

$\mathrm{N}$ April 44 was celebrated, with great ceremony and éclat, the twenty-fifth anniversary of the foundation of the Zoological Station at Naples by Dr. Anton I)ohm. To the general outside public, the eventful day itself was heralded by the appearance in the Bay, just opposite the Zoological Station, of the entire fleet of the station, drawn up in line, and gaily decked with bunting. This consisted of the two steamers, the folumnes Miullor and the Frank Balfour, and five small fishing-boats. In addition, the Italian Government sent a guard of honour in the shape of a second-class cruiser, the Ficrumosic, which remained in attendance all day.

In the Station itself all was excitement and expectancy. In the morning, a deputation consisting of one (ierman one Italian, and one Finglishman, who were supposed to represent the naturalists of each nationality at present working at the Station, waited on Dr. Dohrn, and offered appropriate congratulations, each speaking in the language of his nation. Dr. Dohm, on replying, successfully evaded linguistic difficulties by beginning his speech in German, continuing it in ltalian, and finishing it in English. The same deputation also waited upon and congratulated Dr. Hugo Eisig, Dr. Dohrn's senior assistant, who has been associated with him since the foundation of the Station.

The grand ceremony itself began at two o'clock. The visitors, on arrival, first assembled in the library, and then passed on to the meeting-room, which was situated on the ground floor of the smaller building. This was the largest room available, and it held about one hundred and twenty people. Needless to say, every available seat was occupied. At one end of the room was a small platform and desk, from which the various speakers in turn delivered their discourses. Just above the desk was hung a specially-painted picture, representing the Bay of Naples, and in the foreground a symbolical figure resting against a block of stone, on which was inscribed, "Al l'rof. Dohrn ed ai suoi cooperatori," and the fact of the twenty-fifth anniversary. On a shelf running round the end of the room were arranged the various addresses 